\title{
Overnight variation in tidal expiratory flow limitation in COPD patients and its correction: an observational study
}

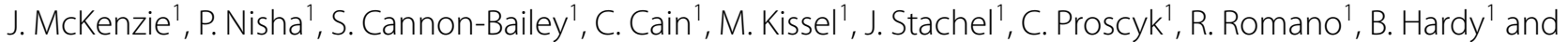 \\ P. M. A. Calverley ${ }^{2,3^{*}}$ (D)
}

\begin{abstract}
Background: Tidal expiratory flow limitation $\left(E F L_{T}\right)$ is common among COPD patients. Whether $E F L_{T}$ changes during sleep and can be abolished during home ventilation is not known.

Methods: COPD patients considered for noninvasive ventilation used a ventilator which measured within-breath reactance change at $5 \mathrm{~Hz}(\Delta \mathrm{Xrs})$ and adjusted EPAP settings to abolish $\mathrm{EFL}_{\mathrm{T}}$. Participants flow limited $(\Delta \mathrm{Xrs}>2.8)$ when supine underwent polysomnography (PSG) and were offered home ventilation for 2 weeks. The EPAP pressure that abolished $\mathrm{EFL}_{\mathrm{T}}$ was measured and compared to that during supine wakefulness. Ventilator adherence and subjective patient perceptions were obtained after home use.

Results: Of 26 patients with supine $\mathrm{EFL}_{T}, 15$ completed overnight PSG and 10 the home study. In single night and 2-week home studies, $\mathrm{EFL}_{T}$ within and between participants was highly variable. This was unrelated to sleep stage or body position with only $14.6 \%$ of sleep time spent within $1 \mathrm{cmH}_{2} \mathrm{O}$ of the awake screening pressure. Over 2 weeks, mean EPAP was almost half the mean maximum EPAP (11.7 vs $6.4 \mathrm{cmH}_{2} \mathrm{O}$ respectively). Group mean $\Delta$ Xrs was $\leq 2.8$ for $77.3 \%$ of their home use with a mean time to abolish new $\mathrm{EFL}_{\mathrm{T}}$ of $5.91 \mathrm{~min}$. Adherence to the ventilator varied between 71 and 100\% in prior NIV users and 36-100\% for naïve users with most users rating therapy as comfortable.

Conclusions: Tidal expiratory flow limitation varies significant during sleep in COPD patients. This can be controlled by auto-titrating the amount of EPAP delivered. This approach appears to be practical and well tolerated by patients.

Trial registration: The trial was retrospectively registered at CT.gov NCT04725500.
\end{abstract}

Keywords: COPD, Noninvasive ventilation (NIV), Tidal expiratory flow limitation (EFL $L_{T}$, Forced oscillation technique, Expiratory positive airway pressure (EPAP)

\section{Background}

Tidal expiratory flow limitation $\left(\mathrm{EFL}_{\mathrm{T}}\right)$ occurs during quiet breathing when flow cannot be increased without an increase in end-expiratory lung volume (EELV). As COPD progresses, tidal expiratory flow limitation $\left(E L_{T}\right)$

*Correspondence: pmacal@liverpool.ac.uk

${ }^{2}$ Institute of Ageing and Chronic Disease, University of Liverpool, Liverpool, UK

Full list of author information is available at the end of the article develops and contributes to dynamic hyperinflation and its associated intrinsic positive end-expiratory pressure $(\mathrm{PEEPi}))$ at rest and during exercise $[1,2]$. The presence of $\mathrm{EFL}_{\mathrm{T}}$ measured when seated is associated with more dyspnoea on exertion [3] and a greater chance of exacerbation and hospitalisation in patients with moderate to severe COPD [4]. The likelihood of developing $\mathrm{EFL}_{\mathrm{T}}$ is markedly increased by postural change when EELV falls $[5,6]$. Lung volume falls further in a state-dependent original author(s) and the source, provide a link to the Creative Commons licence, and indicate if changes were made. The images or other third party material in this article are included in the article's Creative Commons licence, unless indicated otherwise in a credit line to the material. If material is not included in the article's Creative Commons licence and your intended use is not permitted by statutory regulation or exceeds the permitted use, you will need to obtain permission directly from the copyright holder. To view a copy of this licence, visit http://creativecommons.org/licenses/by/4.0/. The Creative Commons Public Domain Dedication waiver (http://creativeco mmons.org/publicdomain/zero/1.0/) applies to the data made available in this article, unless otherwise stated in a credit line to the data. 
fashion with the onset of sleep [7, 8], but to date, we lack data about how this impacts $\mathrm{EFL}_{\mathrm{T}}$.

Noninvasive ventilation (NIV) is an effective strategy for the management of chronic hypercapnic COPD [9]. Different ventilatory approaches have been adopted with high-pressure ventilation proving effective in reducing exacerbations in these sick patients [10]. The application of suboptimal or excessive PEEP may negatively impact adherence to NIV and may result in patient non-compliance [11]. Expiratory flow limitation can be a problem for the ventilated COPD patient and contributes to poor patient-ventilator coordination [12]. Although bronchodilators can abolish $\mathrm{EFL}_{\mathrm{T}}$ in some COPD patients [13], this can be reliably achieved only by the application of sufficient end-expiratory pressure (EPAP) with a resultant decrease in the drive to breathing [14]. Manual pressure titration and clinical judgement usually determine the EPAP which is typically set at a fixed value. However, if $\mathrm{EFL}_{\mathrm{T}}$ is variable, a fixed EPAP pressure may be inadequate or excessive at any given time reducing the clinical benefit of NIV [15]. EFL $\mathrm{T}_{\mathrm{T}}$ can be accurately identified noninvasively using the method of Dellaca et al. based on the within breath change in respiratory system reactance using the forced oscillation technique (FOT) at $5 \mathrm{~Hz}\left(\mathrm{X}_{5}\right)$ [16]. This approach has been adapted to provide continuous monitoring of $\mathrm{EFL}_{\mathrm{T}}$ during noninvasive ventilation [17]. Recently these investigators have shown that changing EPAP can abolish $\mathrm{EFL}_{\mathrm{T}}$ monitored on a breath by breath basis, that this system works in different postures and that it can have physiological benefits in COPD patients studied for one night [18]. However, there is no reported experience with longer periods of ventilatory support using this system.

Before determining whether this new approach is more effective in providing long-term ventilatory support in COPD patients, we conducted an observational study in COPD patients with established tidal expiratory flow limitation when supine, first to determine whether variation in $\mathrm{EFL}_{\mathrm{T}}$ during sleep irrespective of posture and second whether overnight $\mathrm{EFL}_{\mathrm{T}}$ could be abolished by this novel method of ventilatory support in a clinically acceptable way over an extended period.

\section{Methods}

This was a prospective, observational open-label study. Participants were recruited from a convenience sample of patients with a diagnosis of COPD based on standard criteria [19] from two independent pulmonary practices. Participants gave written, informed consent for the study, which was approved by Allendale Investigational Review Board. The study was retrospectively registered at CT.gov NCT04725500. As these were pilot studies, no formal sample size or power calculations were performed.

\section{Participant entry criteria and exclusions}

Participants between the ages of 40 and 80 with a prior COPD diagnosis either using or being considered for ventilatory support were screened for study inclusion. All participants were clinically stable and able to maintain a $\mathrm{SpO} 2$ greater than $88 \%$ at rest and during EPAP titration. Patients with OSA/COPD overlap were also considered eligible provided their OSA was controlled by appropriate inspiratory pressure support. A full list of inclusion and exclusion criteria are provided in the Additional file 1.

\section{Study design}

We used the proprietary ExpiraFlow technology delivered by a noninvasive ventilator (BiPAP A40 EFL, Philips/Respironics, Monroeville, PA) first to determine the presence of $\mathrm{EFL}_{\mathrm{T}}$ awake and then when supine during sleep. In brief, this device uses the forced oscillation methodology to record respiratory system reactance at $5 \mathrm{~Hz}$ during tidal breathing on a breath-by-breath basis, partitioned into inspiratory and expiratory phases as previously described [16]. Further information about the modification of this methodology in this ventilator and details of breath selection and rejection for the calculation of the within-breath reactance change are provided in the online data supplement. We chose a threshold of within-breath reactance change ( $\Delta \mathrm{Xrs}$ ) of more than 2.8 $\mathrm{cmH}_{2} \mathrm{O} / \mathrm{L} / \mathrm{s}$ averaged over 20 artefact-free breaths to identify the presence of $\mathrm{EFL}_{\mathrm{T}}$. When used in ventilatory mode, the BiPAP A40 EFL monitors $\triangle \mathrm{Xrs}$ on a breathby-breath basis. When $\mathrm{EFL}_{\mathrm{T}}$ occurs the ventilator automatically increases the EPAP level until $\mathrm{EFL}_{\mathrm{T}}$ is abolished and PEEPi reduced by applying and adjusting pressure dynamically per-flow limited breath [17] (ExpiraFlow ${ }^{\mathrm{TM}}$ ventilation technology, Philips Respironics). This approach has been shown to substantially reduce the work of breathing in COPD patients breathing spontaneously and with oral-nasal CPAP $[16,20]$.

The study had 3 phases. In the first phase, we identified individuals with $\mathrm{EFL}_{\mathrm{T}}$ when seated and supine using the ExpiraFlow technology with $3 \mathrm{~cm} \mathrm{H}_{2} \mathrm{O}$ EPAP delivered with an oro-nasal mask (Comfort Gel Full Face Mask, Philips Respironics). During the 5-min screening, we needed between 5 and 20 technically valid breaths to determine whether flow limitation was present (see Additional file 1 for details of breath selection) If the presence of flow limitation was confirmed, the ExpiraFlow technology automatically adjusted EPAP to abolish $\mathrm{EFL}_{\mathrm{T}}$ over a $20 \mathrm{~min}$ period of supine breathing and that EPAP value was recorded. The mean pressure needed to overcome $\mathrm{EFL}_{\mathrm{T}}$ in both positions during the titration session was noted as "clinical therapy screening EPAP". 
In the second phase, only participants with $\mathrm{EFL}_{\mathrm{T}}$ when supine in phase 1 underwent a further 20 min session using the ventilator in $\mathrm{S} / \mathrm{T}$ mode with EPAP starting at $4 \mathrm{~cm} \mathrm{H}_{2} \mathrm{O}$ and pressure support of $6 \mathrm{~cm} \mathrm{H}_{2} \mathrm{O}$ together with the FOT. Six $\mathrm{cmH}_{2} \mathrm{O}$ of pressure support was used to ensure that participants had established and $\mathrm{EFL}_{\mathrm{T}}$ and to allow for potential variability in the severity of $\mathrm{EFL}_{\mathrm{T}}$ during the night, thereby avoiding a "floor effect when the EPAP could not be reduced further. Again, the ExpiraFlow technology automatically titrated EPAP until EFL was abolished when awake and supine. If the EPAP that abolished $\mathrm{EFL}_{\mathrm{T}}$ was $\geq 6 \mathrm{cmH}_{2} \mathrm{O}$ in NIV naïve participants or at least equal to the participant's prescribed positive airway pressure (determined outside of the study to address any OSA or oxygenation concerns) for experienced NIV patients, the participant underwent overnight polysomnography (PSG) in a sleep laboratory following current AASM recommendations [21] with the ventilator set in the $\mathrm{S} / \mathrm{T}$ mode as described above. EPAP was automatically adjusted throughout the night to counteract $\mathrm{EFL}_{\mathrm{T}}$. PSG data were initially scored by an automated scoring system (Somnolyzer Philips Respironics) and were verified and scored manually by an accredited RSGPT.

In the third phase, participants completing the PSG were asked if they were willing to use the ventilator each night for a 2-week in-home study. Therapy data were saved on the ventilator's internal memory SD card and were analyzed using Philips proprietary software.

\section{Data analysis and study outcomes}

Tidal expiratory flow limitation was considered to be a binary state. i.e. it was present $\left(\Delta \mathrm{Xrs}>2.8 \mathrm{cmH}_{2} \mathrm{O} / \mathrm{L} / \mathrm{s}\right)$ or absent. EPAP and $\triangle \mathrm{Xrs}$ values were collected and analyzed. The data we report as EPAP is equivalent to automatically titrated PEEPopt [18] i.e. the end-expiratory pressure required to abolish tidal expiratory flow limitation. The overnight variation in $\mathrm{EFL}_{\mathrm{T}}$ was identified by the change in auto-titrated EPAP during sleep while using the ventilator. Abolition of $\mathrm{EFL}_{\mathrm{T}}$ was deemed to be achieved when the average $\Delta \mathrm{Xrs}$ was $<2.8 \mathrm{cmH}_{2} \mathrm{O} / \mathrm{L} / \mathrm{s}$ over a 2 min period.

In phase 2 our primary interest was to determine whether the observed EPAP, our marker of the presence of EFLt, differed during PSG-confirmed sleep from the mean EPAP needed to abolish flow limitation during supine wakefulness. We report data about sleep quality and, where available, the influence of posture and spirometric variables on this outcome. The polysomnographic data were used to determine sleep quality in the single night study and were not related directly to changes in $\mathrm{EFL}_{\mathrm{T}}$.
In phase 3 we wished to establish the minimum, maximum and mean EPAP values during the 2 week study period together with objective adherence data as the average number of hours of treatment use per day and the patients subjective impressions of treatment.

\section{Statistical analysis}

Data are presented as mean and standard deviation or IQR as appropriate. In the single-night study, the percentage of treatment time in which the EPAP pressure averaged over $2 \mathrm{~min}$ was higher, lower or equivalent to the clinical screening EPAP was calculated and presented graphically.

For each participant, the mean values calculated for EPAP are the mean for the days of usage over the two week in-home device use. The mean maximum EPAP value is the mean of the highest EPAP required to abolished EFL on each study night. Additionally, the mean 90\% EPAP value is the mean value of EPAP over the 2-week study period at which the participant spent $90 \%$ of their time at or below. EPAP and $\Delta \mathrm{Xrs}$ values were collected and analyzed to investigate trends using a moving average and linear regression techniques. Sleep quality, sleep staging and body position data are presented using descriptive statistics.

\section{Results}

Participant disposition is summarized in Fig. 1. Fortytwo participants previously diagnosed with mild to severe COPD consented to be in the study. Twenty-three of these participants were prior CPAP or bilevel positive airway pressure users. Twenty-six participants exhibited $E L_{T}$ while supine and four of these participants also exhibited $\mathrm{EFL}_{\mathrm{T}}$ when seated.

Of the 26 participants with $\mathrm{EFL}_{\mathrm{T}}$ while supine, 16 were eligible for the overnight PSG and two week in-home study (56\% male, mean (SD) age 64.9 (6.2) years, BMI 30.3 (7.01). FEV 1 (\% predicted) 47.2 (10.3) and $\mathrm{FEV}_{1} / \mathrm{FVC}$ (\%) 55 (16.9) (Table 1). Fifteen participants agreed to the overnight PSG (Table 1). Ten participants went on to the in-home study. During the in-home study, the two participants with diagnosed obstructive sleep apnoea had their minimum EPAP set to their current CPAP or EPAP settings, if greater than $4 \mathrm{~cm} \mathrm{H}_{2} \mathrm{O}$. The overnight studies did not adjust pressures for any upper airway events.

\section{Single night ventilator study with overnight polysomnography}

Overnight sleep quality varied significantly, as seen in Table 2. One ventilator-naïve participant did not sleep and technical difficulties meant that PSG data from participant 4 were not available. We observed significant between-subject variability in $\mathrm{EFL}_{\mathrm{T}}$. Figure 2 presents 


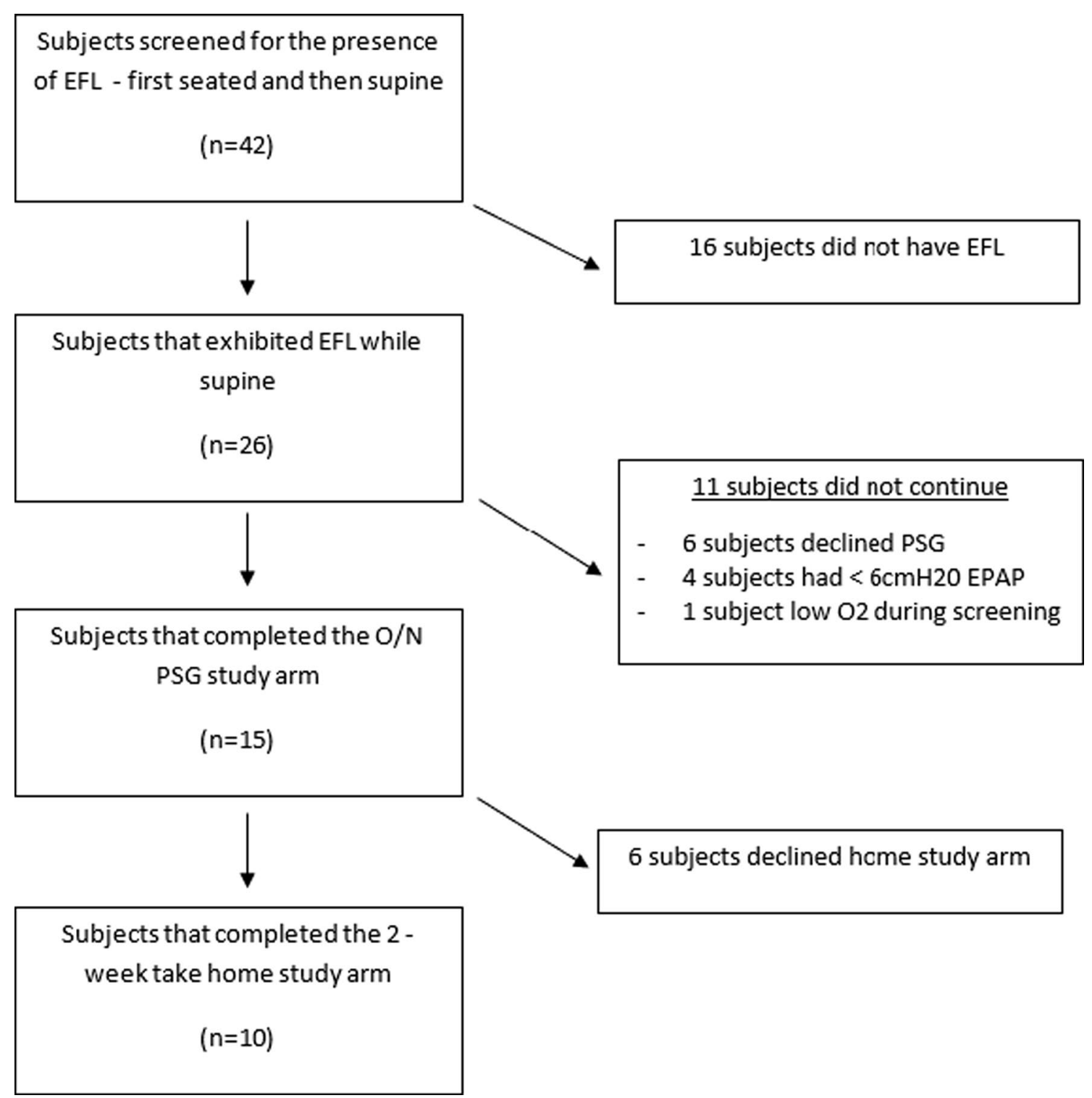

Fig. 1 Study participants flow diagram. * Refers to patient 16 who had $E F L_{T}$ when screened but was unable to complete the PSG study night due to acute illness

the variability of EPAP and $\mathrm{EFL}_{\mathrm{T}}$ throughout the night in four representative cases (participants 8 and 12 naïve to NIV, participants 6 and 11 experienced NIV users) Participants 8 and 11 had $\Delta$ Xrs values close to the 2.8 $\mathrm{cmH}_{2} \mathrm{O} / \mathrm{L} / \mathrm{s}$ threshold throughout the night. In contrast, $\Delta \mathrm{Xrs}$ shows large fluctuations throughout the night in participants 12 and 6 with a similar response by the ExpiraFlow technology to adjust EPAP.

Data for the whole group are shown in Fig. 3 which reflects the EPAP pressure determined during $E_{\mathrm{T}}$ screening (awake and supine) compared to the EPAP pressure determined by the ExpiraFlow technology during the single night overnight PSG. In these participants, on average, only $14.6 \%$ of sleep time was within $\pm 1 \mathrm{cmH}_{2} \mathrm{O}$ of the screening pressure. Fiftyseven percent $(8 / 14)$ of participants spent more than $50 \%$ of their sleep time below their screening EPAP pressure. Approximately 43\% (6/14) of participants spent more than $40 \%$ of their sleep time above the screening pressure.

Table 2 represents total sleep time (TST) and sleep efficiency for overnight sleep study participants. Total sleep time averaged 220 min during the overnight trial with11 sleeping over $180 \mathrm{~min}$. Due to technical issues, 
Table 1 Demographic, physiological and observational data for all subjects that completed the overnight PSG study or the 2-week in-home ventilator use study

\begin{tabular}{|c|c|c|c|c|c|c|c|c|c|}
\hline Participant \# & Sex & Age & BMI & $\begin{array}{l}\text { FEV1 }(\% \\
\text { predicted) }\end{array}$ & FEV1/FVC & Sp02 & $\begin{array}{l}\text { Previous } \\
\text { ventilation user }\end{array}$ & Overnight PSG & $\begin{array}{l}\text { 2-Week } \\
\text { take } \\
\text { home }\end{array}$ \\
\hline 1 & M & 70 & 32.4 & 39 & 52 & 92 & No & Yes & Yes \\
\hline 2 & $\mathrm{~F}$ & 64 & 30.2 & 53 & 68 & 95 & No & Yes & Yes \\
\hline 3 & M & 68 & 30.5 & 48 & 49 & 93 & No & Yes & No \\
\hline 4 & $\mathrm{~F}$ & 78 & 24.8 & 33 & 33 & 95 & No & Yes & No \\
\hline 5 & M & 71 & 38.7 & 32 & 32 & 91 & No & Yes & No \\
\hline 6 & M & 62 & 39.7 & 67 & 86 & 97 & Bilevel & Yes & No \\
\hline 7 & M & 53 & 25.9 & 47 & 61 & 94 & CPAP & Yes & No \\
\hline 8 & $\mathrm{~F}$ & 65 & 27.1 & 56 & 56 & 94 & No & Yes & Yes \\
\hline 9 & $\mathrm{~F}$ & 68 & 35.6 & 43 & 38 & 93 & No & Yes & Yes \\
\hline 10 & M & 69 & 28.9 & 54 & 72 & 93 & Bilevel & Yes & Yes \\
\hline 11 & $\mathrm{~F}$ & 63 & 21.6 & 35 & 45 & 96 & Bilevel & Yes & Yes \\
\hline 12 & M & 56 & 14.5 & 48 & 60 & 90 & No & Yes & Yes \\
\hline 13 & M & 67 & 31.5 & 40 & 52 & 95 & Bilevel & Yes & Yes \\
\hline 14 & M & 64 & 42.3 & 52 & 42 & 92 & Bilevel & Yes & Yes \\
\hline 15 & $\mathrm{~F}$ & 64 & 28.6 & 45 & 45 & 97 & No & Yes & No \\
\hline 16 & $\mathrm{~F}$ & 57 & 33.56 & 65 & 88 & 97 & CPAP & $\mathrm{No}^{*}$ & Yes \\
\hline Average & & 64.9 & 30.3 & 47.2 & 55 & 94 & & & \\
\hline Std. Dev & & 6.2 & 7.01 & 10.3 & 16.9 & 2.1 & & & \\
\hline
\end{tabular}

*This patient had to withdraw from the PSG night due to influenza but was keen to complete the study when they recovered from the acute illness

Table 2 Individual sleep data obtained during the single night polysomnogram

\begin{tabular}{|c|c|c|c|c|c|c|c|c|c|c|c|c|c|c|c|}
\hline Participant ID & 1 & 2 & 3 & 4 & 5 & 6 & 7 & 8 & 9 & 10 & 11 & 12 & 13 & 14 & 15 \\
\hline $\mathrm{AHI}(\mathrm{avg} / \mathrm{h})$ & 26 & 26 & 67.5 & NA & 40.6 & 5.9 & 9 & 3.8 & 1 & 5 & 0 & 12 & 0 & 2 & 19 \\
\hline Time in wake stage (min) & 93 & 38 & 394 & NA & 91 & 263 & 210 & 60 & 294 & 325 & 158 & 219 & 228 & 135 & 208 \\
\hline Time in N1 stage (min) & 22 & 19 & 6 & NA & 30 & 61 & 43 & 43 & 26 & 52 & 14 & 50 & 15 & 7 & 23 \\
\hline Time in N2 stage (min) & 123 & 232 & 2 & NA & 188 & 124 & 145 & 145 & 62 & 54 & 193 & 172 & 94 & 130 & 113 \\
\hline Time in N3 stage (min) & 0 & 0 & 0 & NA & 0 & 6 & 32 & 83 & 35 & 0 & 38 & 0 & 42 & 108 & 0 \\
\hline Time in REM stage (min) & 47 & 126 & 0 & NA & 35 & 3 & 37 & 90 & 78 & 47 & 20 & 48 & 36 & 9 & 14 \\
\hline Total recording time (min) & 285 & 416 & 402 & NA & 345 & 458 & 467 & 421 & 496 & 479 & 423 & 490 & 415 & 391 & 358 \\
\hline Total sleep time (min) & 192 & 377 & 8 & NA & 254 & 195 & 258 & 361 & 202 & 154 & 265 & 270 & 187 & 256 & 150 \\
\hline Sleep efficiency (\%) & $67 \%$ & $91 \%$ & $2 \%$ & NA & $74 \%$ & $42 \%$ & $55 \%$ & $86 \%$ & $41 \%$ & $32 \%$ & $63 \%$ & $55 \%$ & $45 \%$ & $65 \%$ & $42 \%$ \\
\hline
\end{tabular}

Study number represents the same individuals in Table 1. NA not available

PSG data for participant 4 were not available. Sleep efficiency ranged between 2 and 91\%.

The relationship between overnight EPAP and sleep stage was analyzed in 14 of the 15 participants who completed the overnight PSG. From the linear regression trending, four participants experienced an increased median EPAP, and seven experienced a decrease of median EPAP as sleep stage intensity progressed from N1 to REM; three did not show enough EPAP variability to identify trends related to sleep stage.

The relationship between overnight body position and EPAP were analyzed in 9 of the 15 participants who had a body position sensor attached during the PSG. On average, these participants spent $54 \%$ of the night in the supine position, $22 \%$ of the night on their right side, and $21 \%$ on their left side. Although $\mathrm{EFL}_{\mathrm{T}}$ varied as body position changed, the results were not uniform for all participants. Additionally, no relationship was seen between disease severity, whether expressed as $\mathrm{FEV}_{1}$ or $\mathrm{FEV}_{1} / \mathrm{FVC}$ ratio and EPAP variability in the single-night study. 


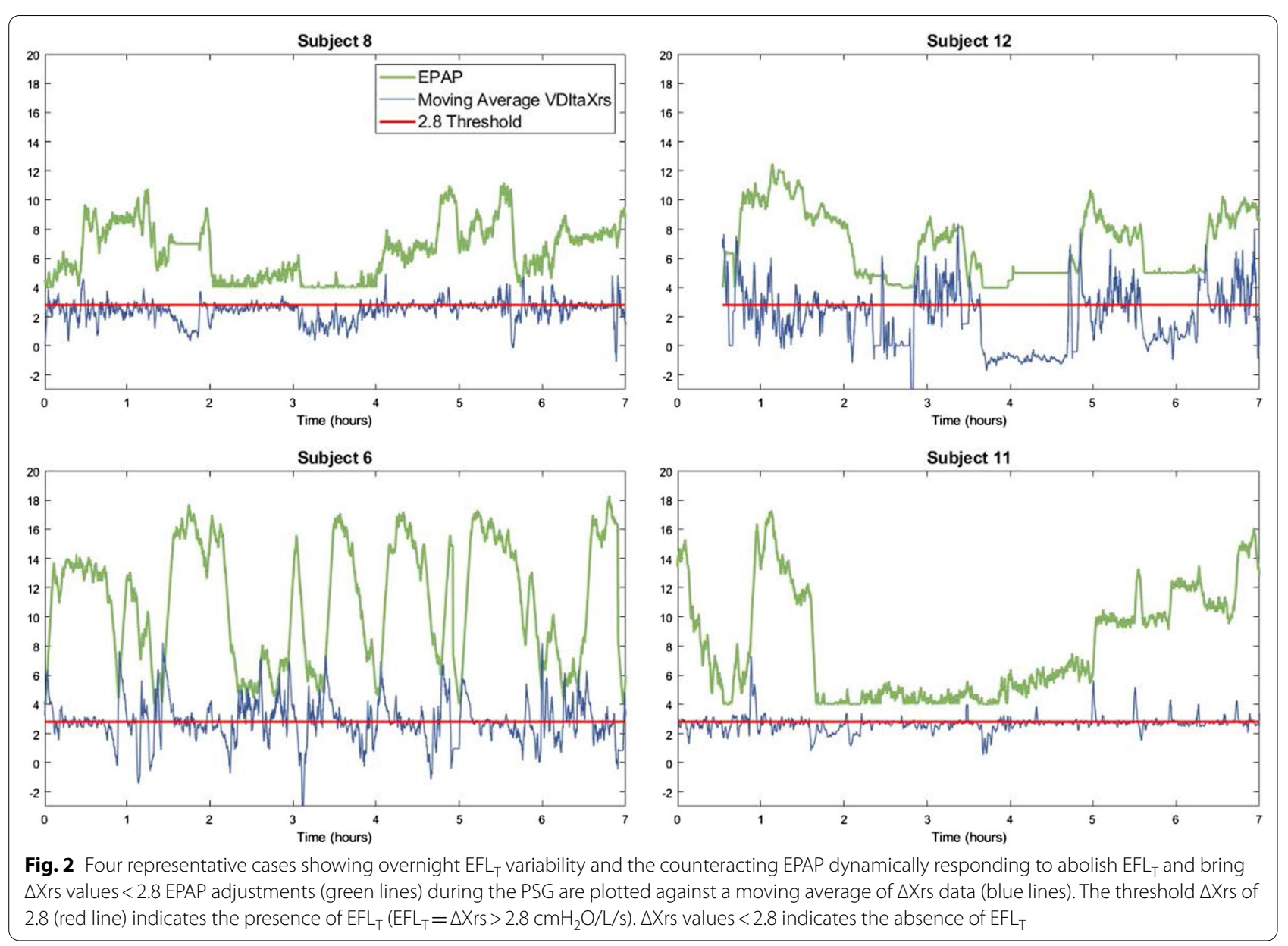

\section{2-Week in-home study}

Ten participants, 4 of whom were ventilator naïve, completed the two week in-home study. Data in Table 3 show the average maximum EPAP value and the average EPAP used for $90 \%$ of the study nights for each participant over the 2 weeks of home use. The variability in EPAP pressure in response to variable $\mathrm{EFL}_{\mathrm{T}}$ is seen by the differences between these average maximum EPAP values for each participant, as well the aggregates for all participants-mean (SD) EPAP (6.4 (1.3) $\mathrm{mH}_{2} \mathrm{O}$ ), the 90\% EPAP (9.3 (3.2) $\mathrm{mH}_{2} \mathrm{O}$ ), and the maximum EPAP (11.7 (3.4) $\mathrm{cmH}_{2} \mathrm{O}$ ). A detailed example of this variability is provided in the data supplement (Additional file 1: Table S1, Fig. S1). There was an inverse relationship between the average EPAP and the $\mathrm{FEV}_{1} / \mathrm{FVC}(\mathrm{r}=-0.68, \mathrm{p}=0.031)$ and to a lesser degree an inverse relationship between average maximum EPAP and FEV1 $(r=-0.55, p=0.103)$ of the participants. A moderate relationship was seen between BMI and mean EPAP $(r=0.59, \mathrm{p}=0.072)$.

Figure 4 shows the distribution of $\Delta \mathrm{Xrs}$ for all participants $\left(77.3 \% \leq 2.8 \mathrm{cmH}_{2} \mathrm{O} / \mathrm{L} / \mathrm{s}\right)$ during their 2-week inhome use. The auto-titration algorithm's effectiveness is shown in Table 4 where the measured minimum, maximum and meanattained $\Delta \mathrm{Xrs}$ values for all participants who completed the 2-week in-home study are presented. The percentage of all values at or below the threshold over the 2-week study period is shown in this table. The percentage of all $\Delta \mathrm{Xrs} \leq 2.8 \mathrm{cmH}_{2} \mathrm{O} / \mathrm{L} / \mathrm{s}$ for all 2-week inhome use participants was $77.3 \%$. In 7 of the 10 patients flow limitation was abolished for $80-100 \%$ of the night but this was not true for 2 subjects with poor ventilator adherence who contributed most of the flow limited data in Fig. 4. $\Delta$ Xrs values $>2.8$ were quickly remedied with a mean time to return $\Delta \mathrm{Xrs} \leq 2.8 \mathrm{cmH}_{2} \mathrm{O} / \mathrm{L} / \mathrm{s}$ of $5.91 \mathrm{~min}$.

Table 5 shows therapy compliance and comfort assessment of each participant in the in-home trial. Naïve participants averaged 3.2 (minimum 1.6, maximum 5.4) hours of daily use with 14-day compliance rate of 57\%. NPPV users averaged $8.4 \mathrm{~h}$ (min 3.03, max 16.3) of daily use with an average compliance rate of 91.4\%. Three out of 4 NPPV users rated FOT therapy very comfortable over their prior NPPV. Survey data were missing for 2 participants. Table 6 breaks down aggregate data for naïve and prior NPPV user groups. 


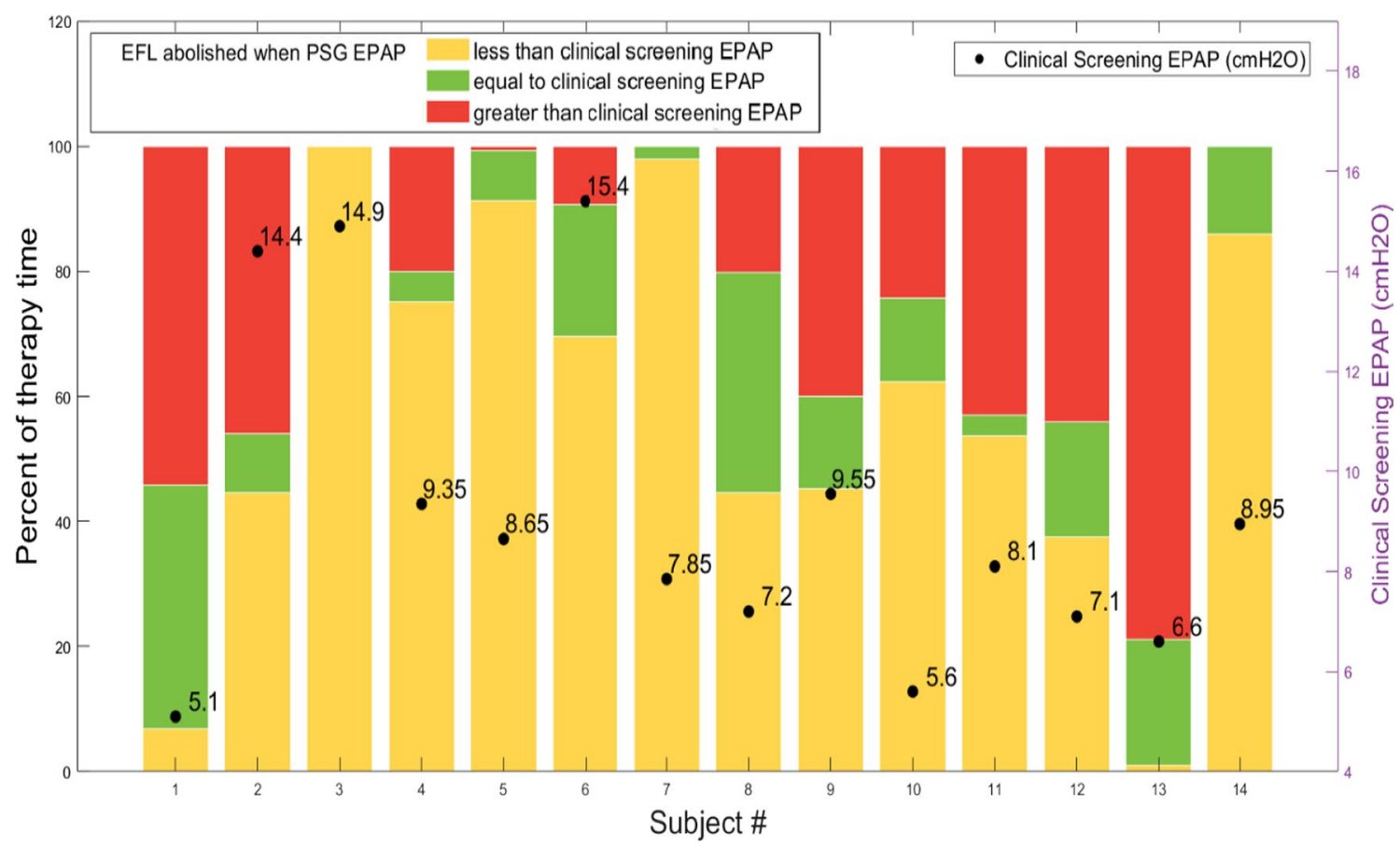

Fig. 3 Percentage of time during PSG that the screening EPAP was equivalent to (green), greater than (yellow) or less than (red) that needed to eliminate $E F L_{T}$ determined when supine awake Black circles and numbers represent the clinical EPAP screening value needed to abolish $E F L_{T}$ (represented on the right-hand Y-axis). Overnight EPAP data were not available for participant 15 due to a data acquisition failure

Table 3 Average EPAP, average max EPAP and average 90\% EPAP for all 2-week in-home study participants—see text for definition of terms

\begin{tabular}{lllll}
\hline Patient ID & Starting EPAP & $\begin{array}{l}\text { Average } \\
\text { Max EPAP }\end{array}$ & Average EPAP & $\begin{array}{l}\text { Average } \\
\mathbf{9 0 \%} \text { EPAP }\end{array}$ \\
\hline 1 & 4.0 & 12.8 & 5.2 & 12.4 \\
2 & 4.0 & 10.5 & 5 & 10.3 \\
8 & 4.0 & 12.1 & 6.8 & 9.7 \\
9 & 4.0 & 12.2 & 7.5 & 10.6 \\
10 & 4.0 & 12.5 & 6.6 & 7.2 \\
11 & 4.0 & 14.7 & 5.6 & 8.6 \\
12 & 4.0 & 6.7 & 5.3 & 5.9 \\
13 & 4.0 & 17.1 & 7.5 & 16.4 \\
14 & 4.0 & 8.1 & 7.9 & 7.9 \\
16 & 4.0 & 10.4 & 4.3 & 7.9 \\
Avg & 4.0 & 11.7 & 6.4 & 9.3 \\
Std Dev & 0.0 & 3.4 & 1.3 & 3.2 \\
\hline
\end{tabular}

Expiratory positive airway pressure is reported in $\mathrm{cmH}_{2} \mathrm{O}$

NPPV user group showed $8.5 \mathrm{~h}$, while naïve group showed $2.5 \mathrm{~h}$. of average nightly therapy use of the device. Altogether with 10 participants, 100 days with $692.4 \mathrm{~h}$. of therapy usage was documented over the two weeks of in-home use.

\section{Discussion}

In this study, we have used a new NIV therapy mode that screens and automatically detects $\mathrm{EFL}_{\mathrm{T}}$ breath by breath using FOT, and dynamically adjusts EPAP to abolish $\mathrm{EFL}_{\mathrm{T}}$ in a population of mild to severe COPD participants. Our principle aim was to investigate whether $\mathrm{EFL}_{\mathrm{T}}$ in patients with $\mathrm{COPD}$ considered for maintenance ventilation varied during sleep and to assess the acceptability of abolishing $\mathrm{EFL}_{\mathrm{T}}$ over a 2 week period. Our main findings were that: (1) $\mathrm{EFL}_{\mathrm{T}}$ was highly variable within and between participants, as seen in overnight studies and over 2-week in-home device use, (2) it was possible to abolish $\mathrm{EFL}_{\mathrm{T}}$ over multiple nights by automatic EPAP adjustment in real time, (3) in overnight PSG studies, on average less than $15 \%$ of sleep time was within $\pm 1 \mathrm{cmH}_{2} \mathrm{O}$ of the screening pressure, (4) sleep quality with the ventilator system was acceptable with an average total sleep time of $220 \mathrm{~min}$, and 11 out of 14 participants sleeping more than $180 \mathrm{~min}$, (5) no direct relationships could be discerned between overnight EPAP and sleep staging or body positioning, and (6) average EPAP for 2-week inhome use participants differed from average $90 \%$ EPAP as well as average max EPAP. These data have implications for the way in which we apply non-invasive ventilation to clinically stable COPD patients. 


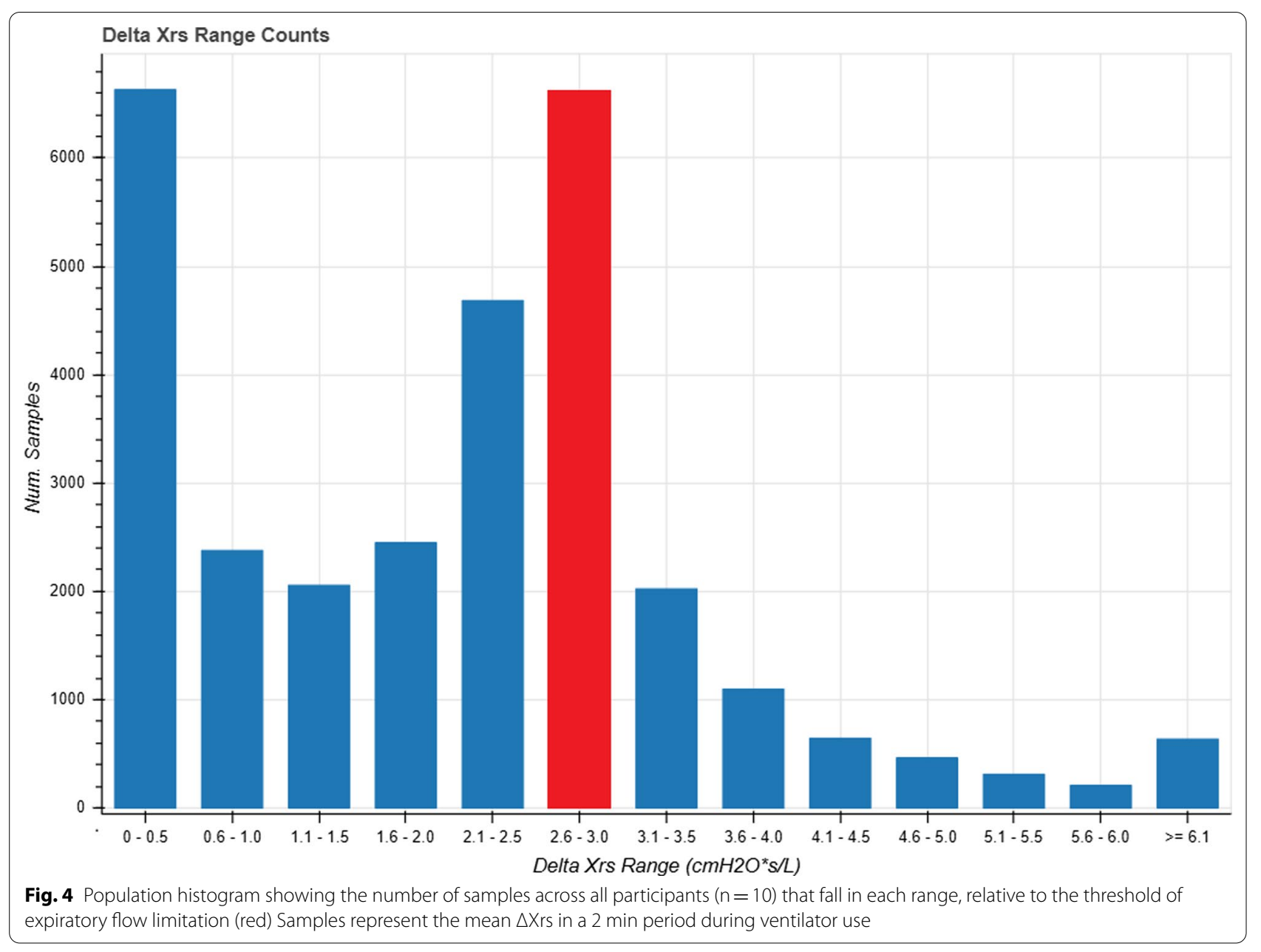

Table 4 Minimum, maximum and average attained $\Delta$ Xrs value for all participants from the 2-week in-home ventilator use

\begin{tabular}{llrll}
\hline Participant \# & $\begin{array}{l}\text { Min } \\
\boldsymbol{\Delta} \text { Xrs }\end{array}$ & $\begin{array}{c}\text { Max } \\
\boldsymbol{\Delta} \text { Xrs }\end{array}$ & $\begin{array}{l}\text { Avg } \\
\boldsymbol{\Delta} \text { Xrs }\end{array}$ & $\begin{array}{l}\text { Percent } \boldsymbol{\Delta \text { Xrs }} \\
<=\mathbf{2 . 8}\end{array}$ \\
\hline 1 & 0.0 & 16.0 & 2.8 & $62.8 \%$ \\
2 & 0.0 & 11.6 & 2.1 & $77.1 \%$ \\
8 & 0.5 & 7.3 & 2.7 & $72.8 \%$ \\
9 & 0.0 & 20.7 & 3.8 & $49.2 \%$ \\
10 & 0.0 & 6.8 & 1.3 & $88.4 \%$ \\
11 & 0.0 & 8.7 & 2.2 & $87.0 \%$ \\
12 & 0.0 & 7.0 & 1.9 & $73.1 \%$ \\
13 & 0.0 & 19.8 & 3.2 & $47.9 \%$ \\
14 & 0.0 & 7.5 & 0.6 & $99.7 \%$ \\
16 & 0.0 & 13.9 & 1.97 & $74.7 \%$ \\
\hline
\end{tabular}

The percent $\Delta$ Xrs $<=2.8$ column shows the percentage of all values at or below the threshold over the 2-week study period
Until recently, it was difficult to determine whether an individual breath was flow limited, let alone multiple breaths. The development of standardized methodologies using the forced oscillation technique [22] and the recognition that the within-breath change in low-frequency respiratory system reactance was highly correlated with more invasive methods of determining the presence of $\mathrm{EFL}_{\mathrm{T}}$ during noninvasive ventilation [17] has transformed this field. We used the novel ExpiraFlow technology to determine dynamically the presence of $\mathrm{EFL}_{\mathrm{T}}$ and the amount of EPAP required to overcome it. Like others $[5,6,23]$ we found that substantially more of our COPD patients developed $\mathrm{EFL}_{\mathrm{T}}$ when supine than erect. Our estimate of the presence of $\mathrm{EFL}_{\mathrm{T}}$ is more conservative than other systems as there was $3 \mathrm{cmH}_{2} \mathrm{O}$ of EPAP present in our ventilator circuit to ensure its effective operation. Moreover, we selected patients who required a significant amount of EPAP to abolish $\mathrm{EFL}_{\mathrm{T}}$ during supine wakefulness. Nonetheless, we saw substantial within and between patient variation in $\mathrm{EFL}_{\mathrm{T}}$, a finding that emphasizes that tidal flow limitation in COPD is not 
Table 5 Therapy compliance and comfort assessment

\begin{tabular}{llccc}
\hline Participant ID & Existing NPPV & Compliance $\%$ & Average night use (h) & NPPV Vs. FOT therapy rating \\
\hline 1 & No & 100 & 2.8 & N/A-Naïve \\
2 & No & 57 & 1.6 & N/A-Naïve \\
8 & No & 36 & 5.4 & N/A-Naïve \\
9 & Yes & 86 & 4.3 & Very comfortable \\
10 & Yes & 100 & 7.9 & Very comfortable \\
11 & Yes & 100 & 10.2 & Very comfortable \\
12 & No & 36 & 2.9 & N/A-Naïve \\
13 & Yes & 100 & 16.3 & Not reported \\
14 & Yes & 71 & 3.03 & Uncomfortable \\
16 & Yes & 0 & 0 & Value missing \\
\hline
\end{tabular}

Table 6 Aggregate therapy compliance and comfort assessment

\begin{tabular}{llll}
\hline Aggregate & Naïve group & NPPV group & Combined \\
\hline Combined days of use & 41 & 69 & 110 \\
Total hours of therapy use & 103.41 & 589.00 & 692.41 \\
Average nightly hours of therapy use & 2.52 & 8.54 & 6.29 \\
\hline
\end{tabular}

a fixed state but one that changes dynamically with body position and during sleep.

The main effect of postural change on $\mathrm{EFL}_{\mathrm{T}}$ is mediated by a fall in end-expiratory lung volume $[6,23]$ something that also occurs in a state-dependent way during sleep [24]. Our data showed marked within night variation in the need for additional EPAP to abolish $\mathrm{EFL}_{\mathrm{T}}$, although the reasons for this are likely to be multifactorial. While the degree of resting airflow obstruction and the body mass index of the patient are potentially important predictors, our single night study group was too small and heterogeneous to confirm this suspicion. A clearer relationship between lung function and the average EPAP needed to abolish $\mathrm{EFL}_{\mathrm{T}}$ was seen in the 2-week data, although the degree to which this varied through the night was not related to waking lung function. Statedependent changes in minute ventilation and breathing pattern are also relevant and may explain why some subjects no longer exhibited $\mathrm{EFL}_{\mathrm{T}}$ when asleep even though it was present during wakefulness. Finally, most of our patients were identified because they developed EFLT when supine. This selection criteria may explain why there was no clear association between posture and EFLT during sleep as the pressure required to overcome flowlimitation related to posture change had already been established. More detailed investigation of these issues across a wider range of COPD patients is needed.

Expiratory flow limitation is an important factor influencing the success of invasive ventilation [12]. Although adjustments in respiratory timing can abolish this when breathing frequency is controlled, only an increase in EPAP can do this during noninvasive ventilation and this must be done without the risk of a consequential increase in end-expiratory lung volume. This is possible using ExpiraFlow technology and is associated with a decrease in respiratory drive measured using the parasternal EMG [14]. Our data extend these observations to patients using this equipment overnight. Sleep quality in COPD patients has been known to be poor for many years [25] and the PSG findings in our single night studies were similar to those in less instrumented patients [26].This was true even though the amount of EPAP applied to abolish $\mathrm{EFL}_{\mathrm{T}}$ increased to $19 \mathrm{cmH} 2$ in some patients. Although the mean EPAP in both the single night and 2-week studies was similar to that anticipated from the awake EPAP setting, this value did not predict the amount of EPAP needed to abolish $\mathrm{EFL}_{\mathrm{T}}$ consistently overnight.

Data from the period of extended use at home showed that $\mathrm{EFL}_{\mathrm{T}}$ was successfully abolished in most patients. Overall treatment adherence was good, with over $6 \mathrm{~h}$ of overnight ventilator use in the 2 weeks of study. However adherence was better in patients who had experience of domiciliary NIV, some of whom used the equipment during waking hours as well. A single night cross over study has suggested that abolishing $\mathrm{EFL}_{\mathrm{T}}$ reduces the number of prematurely initiated breaths during NIV [18]. Other data have shown that neck muscle inspiratory activation during sleep is a 
response to nocturnal hyperinflation and impairs sleep quality [27]. Our data are compatible with a beneficial effect on sleep and treatment adherence by limiting the degree of hyperinflation and sleep disturbance which may underpin the acceptability of treatment. Future studies should explore this possibility.

This observational study has strengths and weaknesses. We identified a subgroup of participants in whom $\mathrm{EFL}_{\mathrm{T}}$ was present when supine and awake. We considered patients attending for initiation or monitoring of treatment, thereby increasing the generalizability of our findings in daily practice. As expected, our volunteers were a mixture of pure COPD and COPD-OSA overlap patients with 2 patients no longer meeting the COPD spirometry criteria when tested before our study. We included their data on an intention to treat basis. We did not see significant differences in the EPAP requirements or the acceptability of ventilatory support between any of these subgroups. However, our data are still best seen as hypothesis-generating. The participants recruited were not severely hypoxemic, and we did not monitor transcutaneous $\mathrm{CO}_{2}$ during sleep or morning arterial blood gas tensions which may have improved with more effective ventilation-perfusion matching across a less flow-limited lung. We did not anticipate the degree to which $\mathrm{EFL}_{\mathrm{T}}$ would change overnight and so more detailed analysis of the degree of the physiological determinants of this process were not incorporated in the study. However, we have identified several areas where further mechanistic investigations are required. We successfully abolished $\mathrm{EFL}_{\mathrm{T}}$ on multiple nights in a 2-week period but not for $100 \%$ of the night. In part this reflects the algorithm we used which adjusts EPAP pressure on a breath-by-breath basis until flow limitation is abolished and hence it takes some time after a change for unrestricted airflow to be restored. However, this was done within 3-6 $\mathrm{min}$ on average without changing the participant's perceived sleep quality.

\section{Conclusions}

Our study has highlighted the spontaneous fluctuation of tidal expiratory flow limitation during sleep, which could contribute to sleep disruption in COPD patients. This can be abolished by the automated application of carefully tailored amounts of EPAP. We have shown that this is both technically possible and clinically acceptable offering the prospect of more physiologically specific forms of noninvasive ventilatory support in spontaneously breathing COPD patients. These proved to be effective and relatively well tolerated with regular use over an extended period of time.

\section{Abbreviations}

BMI: Body mass index; COPD: Chronic obstructive pulmonary disease; CPAP: Continuous positive airway pressure; $\mathrm{DH}$ : Dynamic hyperinflation; $\mathrm{EFL}_{\mathrm{T}}$ : Tidal expiratory flow limitation; FEV1: Forced expiratory volume in the 1st second; FVC: Forced vital capacity; EPAP: Expiratory positive airway pressure; PEEP: Positive end expiratory pressure; PEEPi: Intrinsic positive end expiratory pressure; PEEPopt: Auto-titrated optimal positive end expiratory pressure; FOT: Forced oscillation technique; NIV: Noninvasive ventilation; NPPV: Noninvasive positive pressure ventilation; WOB: Work of breathing; $\triangle \mathrm{Xrs}$ : Difference between the average Xrs measured during inspiration and the one measured during expiration; PSG: Polysomnography; S/T: Spontaneous/timed NIV mode; O/N: Overnight.

\section{Supplementary Information}

The online version contains supplementary material available at https://doi. org/10.1186/s12931-021-01913-7.

Additional file 1: Table S1. Representative case of one participant (participant 11) daily EPAP parameters over 2-weeks. Fig S1. Subject 11 DeltaXrs samples for every session $(n=14)$ for a typical participant (subject 11), where the start of each session treated as time $=0$. Each point represents a 2 min average of the $\Delta$ Xrs values occurring during that time.

\section{Acknowledgements}

Dr. A. J. Cropp and M. Shuntich from Pulmonary Rehabilitation Associates, Youngstown, OH; S. Holbach and J.Tagg from formerly Consolidated Clinical Trials, Inc., Pittsburgh, PA. J. Jasko for Study Data Management, K. Davis and B. Fink for Clinical Study Operations from Philips Respironics.

\section{Authors' contributions}

CC, JM, MK and RR participated in the conception and design of the study. JM, MK, JS and CP were involved with data collection. All authors participated in data analysis and interpretation and were part of preparing the first version of the manuscript. PMAC participated in the interpretation of the data and critically revised the manuscript. All authors read and approved the final manuscript.

\section{Funding}

Philips Respironics was involved with the development of the study protocol, data monitoring, validation, and analysis. The company is the license holder of the technology and provided all equipment (investigational) for the study. Participant recruitment and data collection was the responsibility of the study sites.

Availability of data and materials

Data available on application to the authors.

\section{Declarations}

Ethics approval and consent to participate

Allendale Institutional Review Board approved the study. All participants gave written, informed consent for the study.

\section{Consent for publication}

Not applicable.

\section{Competing interests}

PMAC does not have any directly competing interests but has advised several pharmaceutical companies including GSK, AstraZeneca, Boehringer Ingelheim, Novartis and Recipharm on the conduct and design of clinical trials in COPD. The remaining authors were all employees of Philips Respironics at the time of the conduct of this study.

\section{Author details}

${ }^{1}$ Philips Respironics, Monroeville, PA, USA. ${ }^{2}$ Institute of Ageing and Chronic Disease, University of Liverpool, Liverpool, UK. ${ }^{3}$ University Hospital Aintree, Longmoor Lane, Liverpool L23 8UE, UK. 
Received: 10 July 2021 Accepted: 9 December 2021

Published online: 23 December 2021

\section{References}

1. Calverley PM, Koulouris NG. Flow limitation and dynamic hyperinflation: key concepts in modern respiratory physiology. Eur Respir J. 2005;25:18699. https://doi.org/10.1183/09031936.04.00113204.

2. Krieger BP. Hyperinflation and intrinsic positive end-expiratory pressure: less room to breathe. Respiration. 2009;77(3):344-50.

3. Aarli BB, Calverley PM, Jensen RL, Eagan TM, Bakke PS, Hardie JA. Variability of within-breath reactance in COPD patients and its association with dyspnoea. Eur Respir J. 2015;45:625-34.

4. Aarli BB, Calverley PM, Jensen RL, Dellacà R, Eagan TM, Bakke PS, Hardie JA. The association of tidal EFL with exercise performance, exacerbations, and death in COPD. Int J Chron Obstruct Pulmon Dis. 2017;12:2179-88.

5. Eltayara L, Ghezzo H, Milic-Emili J. Orthopnea and tidal expiratory flow limitation in patients with stable COPD. Chest. 2001;119:99-104.

6. Milesi I, Porta R, Barbano L, Cacciatore S, Vitacca M, Dellacà RL. Automatic tailoring of the lowest PEEP to abolish tidal expiratory flow limitation in seated and supine COPD patients. Respir Med. 2019;155:13-8.

7. Hudgel DW, Devadatta P. Decrease in functional residual capacity during sleep in normal humans. J Appl Physiol Respir Environ Exerc Physiol. 1984;57:1319-22.

8. Avraam J, Dawson A, Rochford PD, Brazzale DJ, O'Donoghue FJ, Trinder J, Jordan AS. The effect of sex and body weight on lung volumes during sleep. Sleep. 2019;42:141.

9. Liao H, Pei W, Li H, Luo Y, Wang K, Li R, Xu L, Chen X. Efficacy of long-term noninvasive positive pressure ventilation in stable hypercapnic COPD patients with respiratory failure: a meta-analysis of randomized controlled trials. Int J Chron Obstruct Pulmon Dis. 2017;12:2977-85.

10. Murphy PB, Rehal S, Arbane G, Bourke S, Calverley PMA, Crook AM, Dowson L, Duffy N, Gibson GJ, Hughes PD, Hurst JR, Lewis KE, Mukherjee R, Nickol A, Oscroft N, Patout M, Pepperell J, Smith I, Stradling JR, Wedzicha JA, Polkey MI, Elliott MW, Hart N. Effect of home noninvasive ventilation with oxygen therapy vs oxygen therapy alone on hospital readmission or death after an acute COPD exacerbation: a randomized clinical trial. JAMA. 2017;317:2177-86.

11. Mansell SK, Cutts S, Hackney I, et al. Using domiciliary noninvasive ventilator data downloads to inform clinical decision-making to optimise ventilation delivery and patient compliance. BMJ Open Respir Res. 2018;5(1): e000238.

12. Junhasavasdikul D, Telias I, Grieco DL, Chen L, Gutierrez CM, Piraino T, Brochard L. Expiratory flow limitation during mechanical ventilation. Chest. 2018;154:948-62.

13. Dellaca RL, Pompilio PP, Walker PP, Duffy N, Pedotti A, Calverley PM. Effect of bronchodilation on expiratory flow limitation and resting lung mechanics in COPD. Eur Respir J. 2009;33(6):1329-37.

14. Suh ES, Pompilio P, Mandal S, Hill P, Kaltsakas G, Murphy PB, Romano R, Moxham J, Dellaca R, Hart N. Autotitrating external positive end-expiratory airway pressure to abolish expiratory flow limitation during tidal breathing in patients with severe COPD: a physiological study. Eur Respir J. 2020;56:1902234.

15. Nava S, Bruschi C, Fracchia C, Braschi A, Rubini F. Patient-ventilator interaction and inspiratory effortduring pressure support ventilation in patients with different pathologies. Eur Respir J. 1997;10(1):177-83.

16. Dellaca RL, Santus P, Aliverti A, et al. Detection of expiratory flow limitation in COPD using the forced oscillation technique. Eur Respir J. 2004;23(2):232-40.

17. Zannin E, Chakrabarti B, Govoni L, Pompilio PP, Romano R, Calverley PMA, Dellacà RL. Detection of expiratory flow limitation by forced oscillations during noninvasive ventilation. Am J Respir Crit Care Med. 2019;200:1063-5.

18. Zannin E, Milesi I, Porta R, Cacciatore S, Barbano L, Trentin R, Fanfulla F, Vitacca M, Dellacà RL. Effect of nocturnal EPAP titration to abolish tidal expiratory flow limitation in COPD patients with chronic hypercapnia: a randomized, cross-over pilot study. Respir Res. 2020. https://doi.org/10. 1186/s12931-020-01567-X.

19. Vogelmeier CF, Criner GJ, Martinez FJ, Anzueto A, Barnes PJ, Bourbeau J, Celli BR, Chen R, Decramer M, Fabbri LM, Frith P, Halpin DM, López Varela
MV, Nishimura M, Roche N, Rodriguez-Roisin R, Sin DD, Singh D, Stockley R, Vestbo J, Wedzicha JA, Agusti A. Global strategy for the diagnosis, management, and prevention of chronic obstructive lung disease 2017 report: GOLD executive summary. Eur Respir J. 2017;49(3):1700214.

20. Dellaca RL, Rotger M, Aliverti A, Navajas D, Pedotti A, Farre R. Noninvasive detection of expiratory flow limitation in COPD patients during nasal CPAP. Eur Respir J. 2006;27(5):983-91.

21. Berry RB, Budhiraja R, Gottlieb DJ, Gozal D, Iber C, Kapur VK, Marcus CL, Mehra R, Parthasarathy S, Quan SF, Redline S, Strohl KP, Davidson Ward SL, Tangredi MM, American Academy of Sleep Medicine. Rules for scoring respiratory events in sleep: update of the 2007 AASM Manual for the Scoring of Sleep and Associated Events. Deliberations of the Sleep Apnea Definitions Task Force of the American Academy of Sleep Medicine. J Clin Sleep Med. 2012;8:597-619.

22. King GG, Bates J, Berger Kl, Calverley P, de Melo PL, Dellacà RL, Farré R, Hall GL, loan I, Irvin CG, Kaczka DW, Kaminsky DA, Kurosawa H, Lombardi E, Maksym GN, Marchal F, Oppenheimer BW, Simpson SJ, Thamrin C, van den Berge M, Oostveen E. Technical standards for respiratory oscillometry. Eur Respir J. 2020;55: 1900753.

23. Uccelli S, Pini L, Bottone D, Ranieri P, Orzes N, Tantucci C. Dyspnea during night-time and at early morning in patients with stable copd is associated with supine tidal expiratory flow limitation. Int J Chron Obstruct Pulmon Dis. 2020;15:2549-58.

24. Koo P, Gartman EJ, Sethi JM, Kawar E, McCool FD. Change in end-expiratory lung volume during sleep in patients at risk for obstructive sleep apnea. J Clin Sleep Med. 2017;13(8):941-7.

25. Calverley PM, Brezinova V, Douglas NJ, Catterall JR, Flenley DC. The effect of oxygenation on sleep quality in chronic bronchitis and emphysema. Am Rev Respir Dis. 1982;126:206-10.

26. Calverley PM, Rennard SI, Clerisme-Beaty E, Metzdorf N, Zubek VB, ZuWallack $R$. Effect of tiotropium on night-time awakening and daily rescue medication usein patients with COPD. Respir Res. 2016;17:27.

27. Redolfi S, Grassion L, Rivals I, Chavez M, Wattiez N, Arnulf I, Gonzalez-Bermejo J, Similowski T. Abnormal activity of neck inspiratory muscles during sleep as a prognostic indicator in chronic obstructive pulmonary disease. Am J Respir Crit Care Med. 2020;201:414-22.

\section{Publisher's Note}

Springer Nature remains neutral with regard to jurisdictional claims in published maps and institutional affiliations.

Ready to submit your research? Choose BMC and benefit from

- fast, convenient online submission

- thorough peer review by experienced researchers in your field

- rapid publication on acceptance

- support for research data, including large and complex data types

- gold Open Access which fosters wider collaboration and increased citations

- maximum visibility for your research: over $100 \mathrm{M}$ website views per year

At BMC, research is always in progress.

Learn more biomedcentral.com/submissions 